\title{
ON THE OSCILLATION OF DIFFERENTIAL TRANSFORMS. IV JACOBI POLYNOMIALS $(1)$
}

\author{
BY \\ G. SZEGÖ
}

1. Introduction. In a paper in the Trans. Amer. Math. Soc. $\left({ }^{2}\right)$, E. Hille proved the following

Theorem A. Let $\alpha \geqq 0, \beta \geqq 0, c \geqq 0$. The differential operation

$$
\vartheta-c=\left(1-x^{2}\right) D^{2}+[\beta-\alpha-(\alpha+\beta+2) x] D-c, \quad D=d / d x,
$$

does not diminish the number of the sign changes in the interval $-1<x<+1$.

More exactly, let $y=y(x)$ be a real-valued non-constant function of $x$, $-1 \leqq x \leqq+1$, with a continuous second derivative (with one-sided derivatives at the end points \pm 1$)$. Then the number of the sign changes of $Y=(\vartheta-c) y$ in $-1,+1$ is not less than that of $y$ in the same interval $\left({ }^{3}\right)$.

First let us observe that under the conditions mentioned $Y$ cannot vanish identically-this being true even for $\alpha>-1, \beta>-1$. More precisely, the solutions of the differential equation $(\vartheta-c) y=0$ which are not identically zero cannot have a continuous second derivative in the closed interval $-1 \leqq x \leqq+1$, provided $c>0$; in the case $c=0$ the solution $y=$ const. is the only one of the kind mentioned $\left.{ }^{4}\right)$. Indeed, let us assume that $c>0$, and let $u(x)$ and $v(x)$ be the solutions of the differential equation mentioned regular at $x=+1$ and $x=-1$, respectively, and satisfying the condition $u(+1)$ $=v(-1)=1[$ see $(2.1)]$. Then by means of the table in $\$ 2$ below we conclude that $u(x)$ and $v(x)$ are linearly independent $\left[u^{\prime}(x) \rightarrow \infty, v^{\prime}(x)=O(1)\right.$ as $x \rightarrow-1+0$ and $u^{\prime}(x)=O(1), v^{\prime}(x) \rightarrow \infty$ as $\left.x \rightarrow 1-0\right]$. Moreover $\left\{c_{1} u(x)\right.$ $\left.+c_{2} v(x)\right\}^{\prime} \rightarrow \infty$ either for $x \rightarrow-1+0$ or for $x \rightarrow 1-0$ (or in both cases) unless $c_{1}=c_{2}=0$.

In the same paper $\mathrm{E}$. Hille proved by means of Theorem A the special case $c=0$ of the following

THEOREM B. Let $\alpha \geqq 0, \beta \geqq 0, c \geqq 0$ and let $\vartheta$ have the same meaning as in

Presented to the Society, February 27, 1943; received by the editors October 17, 1942.

(1) See the previous papers of this series by G. Szegö, E. Hille and A. C. Schaeffer, in the Trans. Amer. Math. Soc. vols. 52, 53 (1942-1943). (Cf. below, loc. cit. footnotes 2 and 6.)

(2) E. Hille, On the oscillation of differential transforms. II. Characteristic series of boundary value problems, Trans. Amer. Math. Soc. vol. 52 (1942) pp. 463-497; see \$2.8.

$\left({ }^{3}\right)$ Regarding the definition of the number of sign changes see, G. Pólya and G. Szegö, Aufgaben und Lehrsätze aus der Analysis, vol. 2, 1925, p. 40.

(4) G. Szegö, Orthogonal polynomials, Amer. Math. Soc. Colloquium Publications, vol. 23, 1939; see p. 61, (4.2.6). 
Theorem A. We denote by $f(x)$ a real-valued function possessing derivatives of all orders in $-1 \leqq x \leqq+1$. If the number of the sign changes of the functions $(\vartheta-c)^{k} f(x), k=1,2,3, \cdots$, is bounded, say at most $N$, then $f(x)$ is a polynomial of degree at most $N$.

The purpose of the present note is to prove

THEOREM A'. Theorem A remains true under the more general condition $\alpha>-1, \beta>-1, c \geqq 0$.

THEOREM B'. Let $\alpha$ and $\beta$ be arbitrary real, $c \geqq 0$. If $f(x)$ satisfies the conditions of Theorem $\mathrm{B}, f(x)$ must be a polynomial of degree at most $N+\gamma$. Here the constant $\gamma=\gamma(\alpha, \beta, c)$ depends only on $\alpha, \beta$ and $c$.

Assuming $\alpha>-1, \beta>-1$, Theorem $\mathrm{B}^{\prime}$ (with $\gamma=0$ ) can be derived from Theorem $\mathrm{A}^{\prime}$ in a manner used first by G. Pólya and N. Wiener in case of Fourier series $\left(^{(5)}\right.$ and applied later to numerous other instances by $\mathrm{E}$. Hille (loc. cit.). We prefer however a direct proof of Theorem $\mathrm{B}^{\prime}$ based on an idea which was used in the first paper of the present series $\left({ }^{6}\right)$.

2. Proof of Theorem $\mathrm{A}^{\prime}$. First we assume $c>0$. Let $u(x)$ be the uniquely determined solution of $(\vartheta-c) y=0$ which is regular at $x=+1$ and for which $u(+1)=1$ holds; we have as well known

$$
\begin{aligned}
u(x) & =F\left(k, k^{\prime} ; l ;(1-x) / 2\right) \\
& =\sum_{n=0}^{\infty} \frac{k(k+1) \cdots(k+n-1) k^{\prime}\left(k^{\prime}+1\right) \cdots\left(k^{\prime}+n-1\right)}{l(l+1) \cdots(l+n-1) \cdot 1 \cdot 2 \cdots n}((1-x) / 2)^{n}
\end{aligned}
$$

where $k$ and $k^{\prime}$ are the roots of the quadratic equation $k(-k+\alpha+\beta+1)=c$ and $l=\alpha+1$. Since $(k+\nu)\left(k^{\prime}+\nu\right)=\nu(\nu+\alpha+\beta+1)+c>0, \nu=0,1,2, \cdots$, we have $u(x)>0$ and $u^{\prime}(x)<0$ in $-1<x \leqq+1$. Incidentally, $k$ and $k^{\prime}$ are different from $0,-1,-2, \cdots ; l>0$.

Let us investigate the behavior of $u(x)$ and $u^{\prime}(x)$ as $x \rightarrow-1+0$. Since

$$
\begin{aligned}
\frac{k(k+1) \cdots(k+n-1) k^{\prime}\left(k^{\prime}+1\right) \cdots\left(k^{\prime}+n-1\right)}{l(l+1) \cdots(l+n-1) \cdot 1 \cdot 2 \cdots n} & \cong \frac{\Gamma(l)}{\Gamma(k) \Gamma\left(k^{\prime}\right)} n^{k+k^{\prime}-l-1} \\
& =\frac{\Gamma(l)}{\Gamma(k) \Gamma\left(k^{\prime}\right)} n^{\beta-1}, n \rightarrow \infty,
\end{aligned}
$$

Cesàro's theorem( $\left.{ }^{7}\right)$ can be applied to $u(x)$ provided $\beta \geqq 0$ and to $u^{\prime}(x)$ provided $\beta>-1$. We obtain the following table:

$\left(^{5}\right)$ G. Polya and N. Wiener, On the oscillation of the derivatives of a periodic function, Trans. Amer. Math. Soc. vol. 52 (1942) pp. 249-256.

(6) G. Szegö, On the oscillation of differential transforms. I, Trans. Amer. Math. Soc. vol. 52 (1942) pp. 450-462.

( $\left.{ }^{7}\right)$ See, for instance, G. Pólya and G. Szegö, Aufgaben und Lehrsätze aus der Analysis, vol. 1,1925 , p. 14 , Problem 85. 


\begin{tabular}{|c|c|c|}
\hline & $u(x) \sim$ & $-u^{\prime}(x) \sim$ \\
\hline$\beta>0$ & $(1+x)^{-\beta}$ & $(1+x)^{-\beta-1}$ \\
\hline$\beta=0$ & $-\log (1+x)$ & $(1+x)^{-1}$ \\
\hline$-1<\beta<0$ & 1 & $(1+x)^{-\beta-1}$ \\
\hline
\end{tabular}

The symbol $f(x) \sim g(x)$ means that $f(x) / g(x)$ approaches a positive limit as $x \rightarrow-1+0$.

We also note the identity

$$
\left\{\begin{array}{l}
Y=(\vartheta-c) y=(1-x)^{-\alpha}(1+x)^{-\beta}\{u(x)\}^{-1} t^{\prime}(x), \\
t(x)=H(x)\left(y^{\prime} u-y u^{\prime}\right), \quad H(x)=(1-x)^{\alpha+1}(1+x)^{\beta+1} .
\end{array}\right.
$$

Now let $y$ have $N$ sign changes in $-1<x<+1, N>0$; then $N$ abscissae $\alpha_{\nu}$ exist, $\alpha_{0}=1>\alpha_{1}>\alpha_{2}>\cdots>\alpha_{N}>\alpha_{N+1}=-1$, such that $y$ is alternately less than or equal to 0 and greater than or equal to 0 in the intervals $\alpha_{\nu+1}, \alpha_{\nu}$ without being identically zero in these intervals. We may assume that in an arbitrary small left-hand neighborhood of $\alpha_{\nu}$ there are abscissae for which $y \neq 0,1 \leqq \nu \leqq N$. (By this condition the $\alpha_{\nu}$ are uniquely determined.) Obviously $y\left(\alpha_{\nu}\right)=0,1 \leqq \nu \leqq N$. Then by Rolle's theorem we conclude the existence of at least $N-1$ zeros for $u^{2}(y / u)^{\prime}=y^{\prime} u-y u^{\prime}$ hence also for $t(x)$ between $\alpha_{1}$ and $\alpha_{N}$ separating the abscissae $\alpha_{\nu}$; in addition $\lim t(x)=0$ as $x \rightarrow 1-0$.

But $t(x)$ must have also a zero in $-1<x<\alpha_{N}$. Assume the contrary, for instance $t(x)<0$ or $(y / u)^{\prime}<0$ in $-1<x<\alpha_{N}$. Then $y / u$ is decreasing in this interval and since $y\left(\alpha_{N}\right)=0$ we must have $y>0$ in $-1<x<\alpha_{N}$ and $y>h u$ in $-1<x \leqq \alpha_{N}-\epsilon\left[0<\epsilon<\alpha_{N}+1, h=h(\epsilon)>0\right]$.

In case $\beta \geqq 0$ we conclude that $y \rightarrow+\infty$ as $x \rightarrow-1+0$ [see table (2.3)] which is a contradiction.

In case $-1<\beta<0$ we obtain $y>h^{\prime}\left(h^{\prime}>0\right)$ for $-1<x \leqq \alpha_{N}-\epsilon$. But in this case $-u / u^{\prime} \sim(1+x)^{\beta+1} \rightarrow 0$ as $x \rightarrow-1+0$ so that

$$
\frac{t(x)}{-(1+x)^{\beta+1} y u^{\prime}}=\frac{(1-x)^{\alpha+1}(1+x)^{\beta+1}\left(y^{\prime} u-y u^{\prime}\right)}{-(1+x)^{\beta+1} y u^{\prime}} \rightarrow 2^{\alpha+1},
$$

hence $t(x)>0$ when $x$ is sufficiently near -1 . This is again a contradiction.

Recapitulating, we have found certain zeros $\beta_{0}, \beta_{1}, \cdots, \beta_{N}$ of $t(x)$ satisfying the inequalities $\beta_{0}=1>\beta_{1}>\cdots>\beta_{N-1}>\beta_{N}>-1$ and $\alpha_{\nu+1}<\beta_{\nu}<\alpha_{\nu}$, $1 \leqq \nu \leqq N$. Repeated application of Rolle's theorem furnishes at least $N$ sign changes of $Y$. Note that $t(x)$ cannot be identically 0 in $\beta_{\nu+1}, \beta_{\nu}$ since this would imply $y / u \equiv$ const., hence $y \equiv 0$ on account of $y\left(\alpha_{\nu+1}\right)=0$. But $y \neq 0$ at suitable points to the left from $\alpha_{v+1}$.

The remaining case $c=0$ can easily be settled. The identity (2.4) holds then with $u(x)=1$, that is, $t(x)=H(x) y^{\prime}$. In this case $t(x)$ has at least $N-1$ zeros in the interior of $-1,+1$ and in addition the zeros $x= \pm 1$. 
3. Proof of Theorem $B^{\prime}$. Let us start with certain preliminary remarks on Jacobi polynomials $P_{n}^{(\alpha, \beta)}(x)$. For arbitrary real values of $\alpha$ and $\beta$ we use the definition [see Szegö, loc. cit. $\left({ }^{4}\right)$ p. $61,(4.21 .2)$ ]

$$
\left\{\begin{aligned}
& P_{0}^{(\alpha, \beta)}(x)=1 ; \\
& P_{n}^{(\alpha, \beta)}(x)=C_{n+\alpha, n} F(-n, n+\alpha+\beta+1 ; \alpha+1 ;(1-x) / 2) \\
&=(n !)^{-1} \sum_{\nu=0}^{n} C_{n, \nu}(n+\alpha+\beta+1) \cdots(n+\alpha+\beta+\nu)(\alpha+\nu+1) \\
& \cdots(\alpha+n)((x-1) / 2)^{\nu}, \quad n \geqq 1 .
\end{aligned}\right.
$$

Then $y=P_{n}^{(\alpha, \beta)}(x)$ satisfies the differential equation $(\vartheta+n(n+\alpha+\beta+1)) y=0$ [Szegö, loc. cit. p. 59, (4.2.1)]. Furthermore, except for an additive constant [loc. cit. p. 62, (4.21.7)]

$$
\int P_{n}^{(\alpha, \beta)}(x) d x=2(n+\alpha+\beta)^{-1} P_{n+1}^{(\alpha-1, \beta-1)}(x) .
$$

We also note Rodrigues' formula [loc. cit. p. 66, (4.3.1)]

$$
\begin{aligned}
& (1-x)^{\alpha}(1+x)^{\beta} P_{n}^{(\alpha, \beta)}(x) \\
& \quad=(-1)^{n}\left(2^{n} n !\right)^{-1}(d / d x)^{n}\left\{(1-x)^{n+\alpha}(1+x)^{n+\beta}\right\} .
\end{aligned}
$$

From (3.1) we see that $P_{n}^{(\alpha, \beta)}(x), n \geqq 1$, is of the precise degree $n$ provided $\alpha+\beta \neq-2,-3,-4, \cdots$. If $\alpha+\beta=-l-1, l$ positive integer, $P_{n}^{(\alpha, \beta)}(x)$ is still of the precise degree $n$ provided $n>l$.

In case $\alpha>-1, \beta>-1$ we conclude from (3.3) in the familiar manner the orthogonality relation

$$
\int_{-1}^{+1}(1-x)^{\alpha}(1+x)^{\beta} P_{n}^{(\alpha, \beta)}(x) q(x) d x=0
$$

where $q(x)$ is an arbitrary polynomial of degree $n-1$. Now let $\alpha$ and $\beta$ be arbitrary real and let $m$ be the smallest non-negative integer such that $\alpha+m>-1, \beta+m>-1$. Taking $n \geqq 2 m+1$ and $q(x)=\left(1-x^{2}\right)^{m} r(x)$ where $r(x)$ is an arbitrary polynomial of degree $n-2 m-1$ we find that for this particular type of polynomials $q(x)$ the orthogonality relation (3.4) still holds.

Under the same condition we have [loc. cit. p. 62, (4.21.6), p. 67, (4.3.3)]

$$
\begin{aligned}
\int_{-1}^{+1}(1-x)^{\alpha+m} & (1+x)^{\beta+m} P_{n}^{(\alpha, \beta)}(x) x^{n-2 m} d x \\
= & (-1)^{m} 2^{n+\alpha+\beta+1} \frac{\Gamma(n+\alpha+1) \Gamma(n+\beta+1)}{\Gamma(2 n+\alpha+\beta+2)} \neq 0 .
\end{aligned}
$$


After these preliminaries we proceed to the proof of Theorem $\mathbf{B}^{\prime}$. First let us exclude the case $\alpha+\beta=-l-1, l$ positive integer. We expand $f^{(m)}(x)$ in a series of Jacobi polynomials $P_{n}^{(\alpha+m, \beta+m)}(x)$ :

$$
f^{(m)}(x)=\sum_{n=0}^{\infty} f_{n} P_{n}^{(\alpha+m, \beta+m)}(x) .
$$

Term-by-term integration and use of (3.2) furnishes

$$
\begin{array}{r}
f(x)=\phi(x)+\sum_{n=0}^{\infty} 2^{m}\{(n+\alpha+\beta+2 m)(n+\alpha+\beta+2 m-1) \\
\cdots(n+\alpha+\beta+m+1)\}^{-1} f_{n} P_{n+m}^{(\alpha, \beta)}(x)
\end{array}
$$

where $\phi(x)$ is a polynomial of degree $m-1$ [for $m=0$ we have $\phi(x)=0$ ]. Since in this case $P_{n}^{(\alpha, \beta)}(x)$ is of the precise degree $n$ we can write

$$
f(x)=\sum_{n=0}^{\infty} \phi_{n} P_{n}^{(\alpha, \beta)}(x) \text {. }
$$

Obviously

$$
(\vartheta-c)^{k} f(x)=\sum_{n=0}^{\infty}(-1)^{k}[c+n(n+\alpha+\beta+1)]^{k} \phi_{n} P_{n}^{(\alpha, \beta)}(x) .
$$

Now let $k$ belong to a certain infinite sequence such that the corresponding functions $(\vartheta-c)^{k} f(x)$ have a fixed number, $M$ say, sign changes; $M \leqq N\left({ }^{8}\right)$. We denote the abscissae at which these sign changes take place by $x_{1}, x_{2}, \cdots, x_{M}$; $x_{\nu}=x_{\nu}(k)$. Then if $\delta=+1$ or -1 is properly chosen,

$$
\begin{array}{r}
\delta \int_{-1}^{+1}(1-x)^{\alpha+m}(1+x)^{\beta+m}\left\{(\vartheta-c)^{k} f(x)\right\}\left(x-x_{1}\right) \\
\cdots\left(x-x_{M}\right)\left(1 \pm x^{p}\right) d x>0 .
\end{array}
$$

Here $\rho$ is an arbitrary non-negative integer and $\delta$ does not depend on $\rho$. Substituting for $(\vartheta-c)^{k} f(x)$ its expansion (3.9) the arising integrals will all vanish provided $n>2 m+M+\rho$. However for $n=n^{\prime}=2 m+M+\rho$ we obtain $\pm \delta(-1)^{k}\left[c+n^{\prime}\left(n^{\prime}+\alpha+\beta+1\right)\right]^{k}$

$$
\cdot \phi_{n^{\prime}} \int_{-1}^{+1}(1-x)^{\alpha+m}(1+x)^{\beta+m} P_{n^{\prime}}^{(\alpha, \beta)}(x) x^{M+\rho} d x
$$

and the last integral is different from 0 because of (3.5). Hence if $\phi_{n^{\prime}} \neq 0$ we find for $k \rightarrow \infty$

$$
\left[c+n^{\prime}\left(n^{\prime}+\alpha+\beta+1\right)\right]^{k}=O(1) \max _{0 \leqq \nu \leqq n^{\prime}-1}|c+\nu(\nu+\alpha+\beta+1)|^{k}
$$

(8) From here on we use the argument of the paper cited in footnote 6 . 
which is impossible provided

$$
\left|c+n^{\prime}\left(n^{\prime}+\alpha+\beta+1\right)\right|>\max _{0 \leqq \nu \leqq n^{\prime}-1}|c+\nu(\nu+\alpha+\beta+1)| .
$$

This is the case if $n^{\prime} \geqq n_{0}=n_{0}(\alpha, \beta, c)$.

The previous argument furnishes $\phi_{n}=0$ for $n \geqq 2 m+M, n \geqq n_{0}$, which is equivalent to the assertion of Theorem $\mathrm{B}^{\prime}$.

In case $\alpha+\beta=-l-1, l$ positive in teger, this proof needs a slight modification. We integrate then only the terms $n \geqq m+1$ in (3.6) and conclude (3.7) with the modification that the summation is now extended over the range $n \geqq m+1$ and $\phi(x)$ is a polynomial of degree $2 m$. [The expression in the braces of (3.7) is then positive since $2 m+\alpha+\beta+2>0$.] As a further addition to the previous argument we have to show that

$$
(\vartheta-c)^{k} \phi(x)=O(1)\left|c+n^{\prime}\left(n^{\prime}+\alpha+\beta+1\right)\right|^{k}, \quad k \rightarrow \infty,
$$

uniformly for $-1 \leqq x \leqq+1$ provided $n^{\prime}$ is sufficiently large, $n^{\prime} \geqq n_{1}=n_{1}(\alpha, \beta, c)$. But $(\vartheta-c)^{k} \phi(x)$ is a polynomial of degree $2 m$ and the last assertion follows if we can show that the coefficients of this polynomial have moduli at most $R S^{k}$; here $R>0$ depends on $f(x), \alpha, \beta, c$ and $S>0$ depends only on $\alpha, \beta, c$. Now

$$
\begin{aligned}
(\vartheta-c) x^{h}= & h(h-1)\left(1-x^{2}\right) x^{h-2} \\
& +h[\beta-\alpha-(\alpha+\beta+2) x] x^{h-1}-c x^{h}
\end{aligned}
$$

hence with arbitrary constants $\lambda_{h}$

$$
(\vartheta-c) \sum_{h=0}^{2 m} \lambda_{h} x^{h} \ll S \cdot \max \left|\lambda_{h}\right| \cdot \sum_{h=0}^{2 m} x^{h}
$$

where

$$
S=2 \cdot 2 m(2 m-1)+2 m|\beta-\alpha|+2 m|\alpha+\beta+2|+|c| .
$$

This furnishes the statement by taking for $R$ the maximum modulus of the coefficients of $\phi(x)$ and choosing $S$ according to (3.13).

Theorems $B$ and $B^{\prime}$ remain of course true if the condition regarding $(\vartheta-c)^{k} f(x)$ is satisfied only for an infinite number of values of $k$.

STANFORD UNIVERSITY,

Stanford University, Calif. 